\title{
Research Paper: Alterations in Balance Strategies Due to Joint Bracing in Static Postural Standing
}

\author{
Mohammed Ashtiani ${ }^{1,2}$ (D, Mahmood-Reza Azghani ${ }^{* *}$ (iD) \\ 1. Faculty of Biomedical Engineering, Sahand University of Technology, Tabriz, Iran. \\ 2. Department of Physiotherapy, Faculty of Medical Sciences, Tarbiat Modares University, Tehran, Iran
}

$\begin{gathered}\text { Use your device to scan } \\ \text { and read the artice online }\end{gathered}$
of Modern Rehabilitation. 2018; 12(3):149-156. http://dx.doi.org/10.32598/JMR.V12.N3.149
dol http://dx.doi.org/10.32598/JMR.V12.N3.149

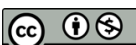

Article info:

Received: 23 Dec 2017

Accepted: 10 May 2018

Available Online: 01 Jul 2018

Keywords:

Posture, Postural balance, Braces, Computer simulation

\begin{abstract}
Introduction: Employment of braces can increase the standing stability by external support of the joints. Examining the effects of different braces, specifically in numerous static postures is practically difficult; therefore, the current parametric study aimed at modeling the alterations of the muscular recruitment patterns during static postures caused by wearing different braces.

Materials and Methods: A biomechanical model of the musculoskeletal system was employed to simulate over 27,000 standing postures with different joint angles and brace conditions. Activation of the muscles in standing posture was calculated using optimized inverse dynamics. The postures with muscle efforts beyond the physiological limit were considered as the feasible postures.

Results: Braces increased the number of feasible postures. However, their effects depended on the joint angles. Knee flexion assisted in providing balance for more postures. The maximum number of feasible postures occurred in midrange knee flexion (30 degrees). High and low stiffness braces caused relatively similar outcomes. Wearing the upper joint braces (knee or hip) relieved the ankle joint muscles and prominently recruited the knee muscles to maintain the balance. Results also suggested that if an individual, in practice, intended to wear only one brace to have the most efficient support in balance, the ankle brace was the best choice.
\end{abstract}

Conclusion: Braces can assist in providing balance for the quiet standing condition. It is better to let knee joint rotate and support the ankle by braces.

\section{Introduction}

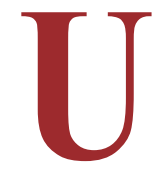

pright standing is the vital task of the human musculoskeletal system. Keeping the balance allows the individuals to do their routine daily activities [1]. Muscles, under the commands of central nervous system, provide internal resistive forces against the external disturbances such as the body weight. In addition to the possible interference with the controlling factors such as balance feedback from vision, vestibular, and somatosensory, there are some deficits in the musculoskeletal components that may reduce the stability of the body [2-6]. For instance, weakness of the muscles, in-

* Corresponding Author:

Mahmood-Reza Azghani, PhD.

Address: Faculty of Biomedical Engineering, Sahand University of Technology, Tabriz, Iran.

Tel: +98 (413) 3458417

E-mail:azghani@sut.ac.ir 
juries in supportive soft tissues, arthritis, etc. may affect the postural balance. Medical interventions, such as joint replacements and employment of assisting devices or orthoses, aimed at healing these disorders may also change the strategies during maintenance of the posture [7-13] .

Employment of the braces, straps, or tapes successfully relieve the pain or healing due to unloading the injured joint, muscle, or connective tissue, and also, increased proprioceptive acuity by providing external stiffness [14]; however, their consequences may limit the body locomotion due to immobilization or reduction in the motion range. The importance of this point is more highlighted due to the postural adjustment, i.e. changing the joints position to reach an alternate balanced posture [1517].

The literature showed that wearing semi-rigid braces in order to immobilize the joint leads to postural instability. Jeon et al. (2013) examined the effects of the ankle and knee immobilization on path length and total time of center of pressure movement in clinical standing tests. They found that wearing an ankle rigid brace restricts the postural reactions during standing [18]. Furthermore, de Freitas et al. (2009) measured the center of pressure of subjects with knee, hip, and trunk joint constraints in addition to the free-to-move condition and concluded that the latter case revealed higher stability [19].

Employment of a biomechanical modeling can confer several advantages to elucidate the effects of brace location and stiffness of the postural balance features. Models can reduce the cost and problems of the practical experiments, specifically when the number of testing conditions is high. Besides, some theoretical cases are difficult to perform in the experiments. For instance, it is arduous to pose a subject in a fixed posture.

The models, however, can analyze these difficult theoretical conditions that can reveal valuable outcomes after assuring its validity. The literature employed parametric modeling to evaluate balance parameters in numerous static postures to assess the pure effect of posture kinematics [20-22] and aging [23] on joint moments or muscle activations.

Although few studies investigated the role of joint immobilization, it was found that free joint condition caused higher levels of stability in upright standing [12, 18, 19]. The current study, however, tried to numerically analyze the standing of a biomechanical model that used different joint braces. The study aimed at obtaining muscle activation or biomechanical joint contributions in a quiet standing model wearing joint braces with different stiffness. It was hypothesized that the employment of the moderately-stiff braces can assist the muscles to pose the body in more feasible postures in the sagittal plane.

\section{Materials and Methods}

\section{Model}

A biomechanical model of human musculoskeletal system was employed from a commercial code (Anybody, version 5, Aalborg, Denmark). The standing model consisted of 44 Hill-based muscles with precise insertions and origins relative to the joints. The code used optimization to find the redundant muscle forces during simple standing in the sagittal plane by minimizing the sum of squared activation, subjected to the equilibrium condition. The input to the standing model was the synthesized series of joint angles (ankle, knee, and hip). Inverse dynamics was utilized to calculate the unknown muscle forces (activation).

One thousand different postures (10 angles of the ankle $\times 10$ angles of the knee $\times 10$ angles of the hip) were defined to be valued in $0,10,20, \ldots, 80,90$ flexion degrees. Each posture was solved in 27 brace combinations (three stiffness levels of ankle brace $\times$ three for knee $\times$ three for hip). The stiffness levels were no stiffness $\left(\mathrm{s}_{0}=0\right)$, low stiffness (similar to a torsional spring with stiffness, $\left.\mathrm{s}_{1}=20 \mathrm{Nm} / \mathrm{rad}\right)$ and high stiffness $\left(\mathrm{s}_{2}=40 \mathrm{Nm} / \mathrm{rad}\right)$. Therefore, totally 27,000 unique cases of brace-worn static postures were produced. Figure 1 shows the flowchart of the simulation.

These values were calculated for a simplified shell model of braces based on linear elastic modulus of neoprene as 1.5 MPa [24]. The linear stiffness (k) was assessed by hand calculations as $\mathrm{k}=\mathrm{EA} / \mathrm{l}$ where $\mathrm{A}$ is area (thickness of the brace) and 1 is its length. The torsional stiffness, then, was determined using equation $\mathrm{K}=\mathrm{kr}^{2}$, where $r$ is the average distance if the brace fibers from the joint. The guessing parameters were $A=4.7 \mathrm{~cm}^{2}, \mathrm{l}=15$ $\mathrm{cm}, \mathrm{r}=7.5 \mathrm{~cm}$. The torsional stiffness was $26 \mathrm{Nm} / \mathrm{rad}$. It was finally rounded to 20 and considered a stiffer one as $40 \mathrm{Nm} / \mathrm{rad}$ with two levels of the stiffness, in addition to the no brace condition.

\section{Feasibility}

The feasibility of standing in different postures and brace conditions was determined after the analysis. In the cases that at least one muscle exceeded $95 \%$ of its maximum force generation limit were labeled as infea- 


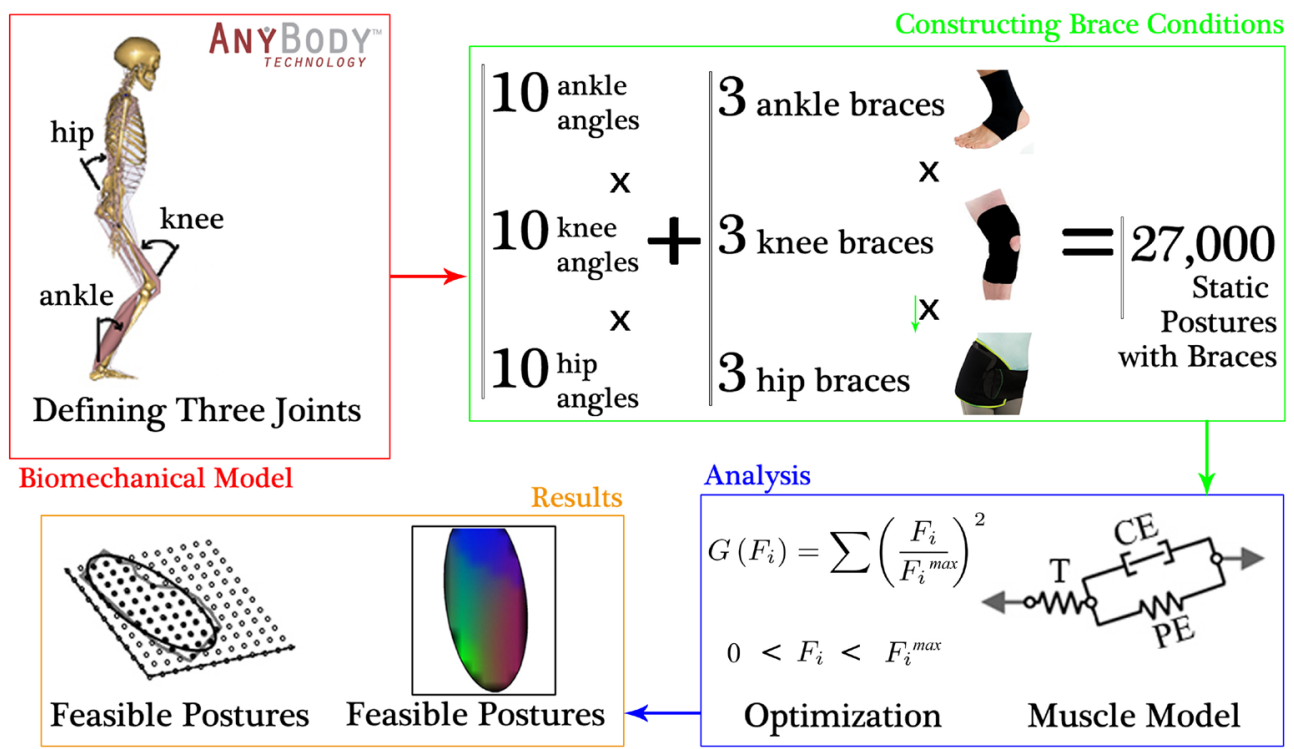

Figure 1. Chematic representation of the simulation steps

Terms and abbreviations: $\mathrm{G}=$ Cost function; $\mathrm{F}_{\mathrm{i}}=$ Muscle forces; $\mathrm{N}_{\mathrm{i}}=$ Muscle areas; $\mathrm{F}_{\mathrm{i}} \max =$ Maximum muscle forces; $\mathrm{T}=$ Tendon force component; $\mathrm{CE}=$ Contractile Element force component; $\mathrm{PE}=$ Passive Element force component

sible. It meant that the feasible postures were the ones with muscles all in their physiological range. Also, the ankle flexion more than 35 degrees was omitted from the outputs due to the sole heel off [25].

\section{Joint contributions}

Muscular contribution of the joints was also determined by division of sum of squared activation of jointrelated muscles by sum of squared activation of all muscle activation, or where $\mathrm{j}$ denoted the joints and $\mathrm{i}$ was the muscles acting on the joint $j$ Further details were presented in references [22, 23].

$$
J C_{j}=\frac{\sum_{i} \alpha_{i}{ }_{i}}{\sum_{j} \sum_{i} \alpha^{2}}
$$

\section{Results}

Figure 2 shows the feasible regions of standing in hip-ankle coordination. Two knee angles (10 and 30 degrees) are selected to represent the results. Among 27 different combinations of the braces, six cases were opted to make the outcomes comparable.

Employment of the ankle braces extended the feasible region along the ankle direction. Relatively, the same results existed for the hip brace application. In greater knee flexion, due to the vast region of the feasible postures, the effects of the braces were not well-observed.
Once the model theoretically used the braces for all joints, the feasible postures decreased even with the low stiffness braces.

The area trapped between the feasible postures was considered as a measure for the number of feasible postures plotted against the knee flexion for the beforementioned six brace conditions. Roughly, all brace wearing cases had the maximum feasible postures near midrange knee flexion angles, i.e. 40 to 50 degrees. The lowest area in knee flexion belonged to the no brace case or fully unsupported model.

The highest area through the knee angles was also possessed by the high stiffness for all joints. In contrast to the early knee angles (as shown in Figure 3) in which bracing all joints slightly reduced the feasible area, the fully supported model by the straps had the greatest feasible ellipse, specifically after 30 -degree knee flexion.

Contribution of the joint mechanisms based on the muscular activation around a joint is illustrated in Figure 4. Since there are three joint mechanisms here, the red-green-blue spectrum is used to show the co-work between the joint mechanisms. In this way, the magenta color denotes the same co-work between the ankle (red) and the hip (blue) strategies. In 10 degrees knee flexion, the red spectrum (ankle) is prominent unless the hip brace usage existed. It is observed that the knee becomes the overriding joint contribution, while the model used 


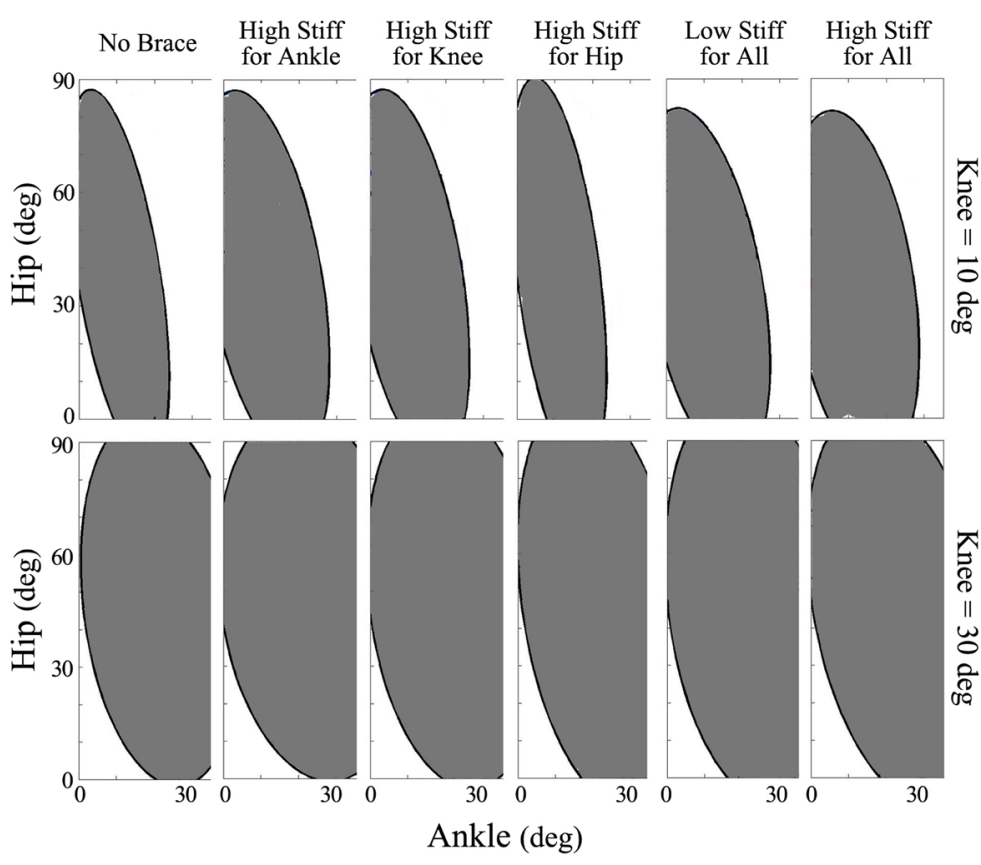

Figure 2. Analogous ellipses for the feasible standing areas in constant knee angles for various bracing conditions

upper limb braces. By flexion of the knee to 30 degrees, wearing the lower limb braces is led to role-playing of the hip joint, specifically in flexed hip postures. But the knee becomes the reliable mechanism in equilibrium when the hip brace is worn.

\section{Discussion}

The current study analyzed the role of the braces on the lower limb joints with different stiffness using a numerical model in various quiet standing postures. The braces were modeled by torsional springs attached to the ankle, knee, and hip in two levels of stiffness. The current study aimed at obtaining the changes in the activation of mus- cles due to wearing braces to determine the reallocation of muscles' effort in provision of balance.

Employing such a parametric modeling elucidated the main and interactive effects of two factors, i.e. the postural kinematics, and the brace wearing. Solving 27,000 cases of static balance in order to find the muscle recruitment patterns unveiled that the contribution of muscles at a certain joint known as joint mechanisms were not directly-influenced by the kinematics. This finding was consistent with those of the previous works that used similar methodology [21-23]. The literature, on the other hand, uttered that rigid bracing can reduce the stability during quiet standing $[18,19]$, but they mostly used the

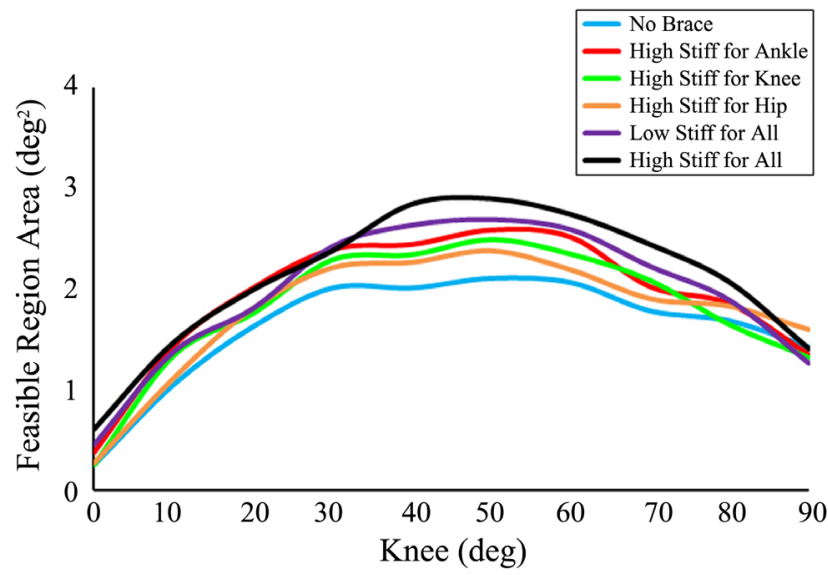

Figure 3. Area of the feasible regions against the knee flexion angles for different bracing conditions 


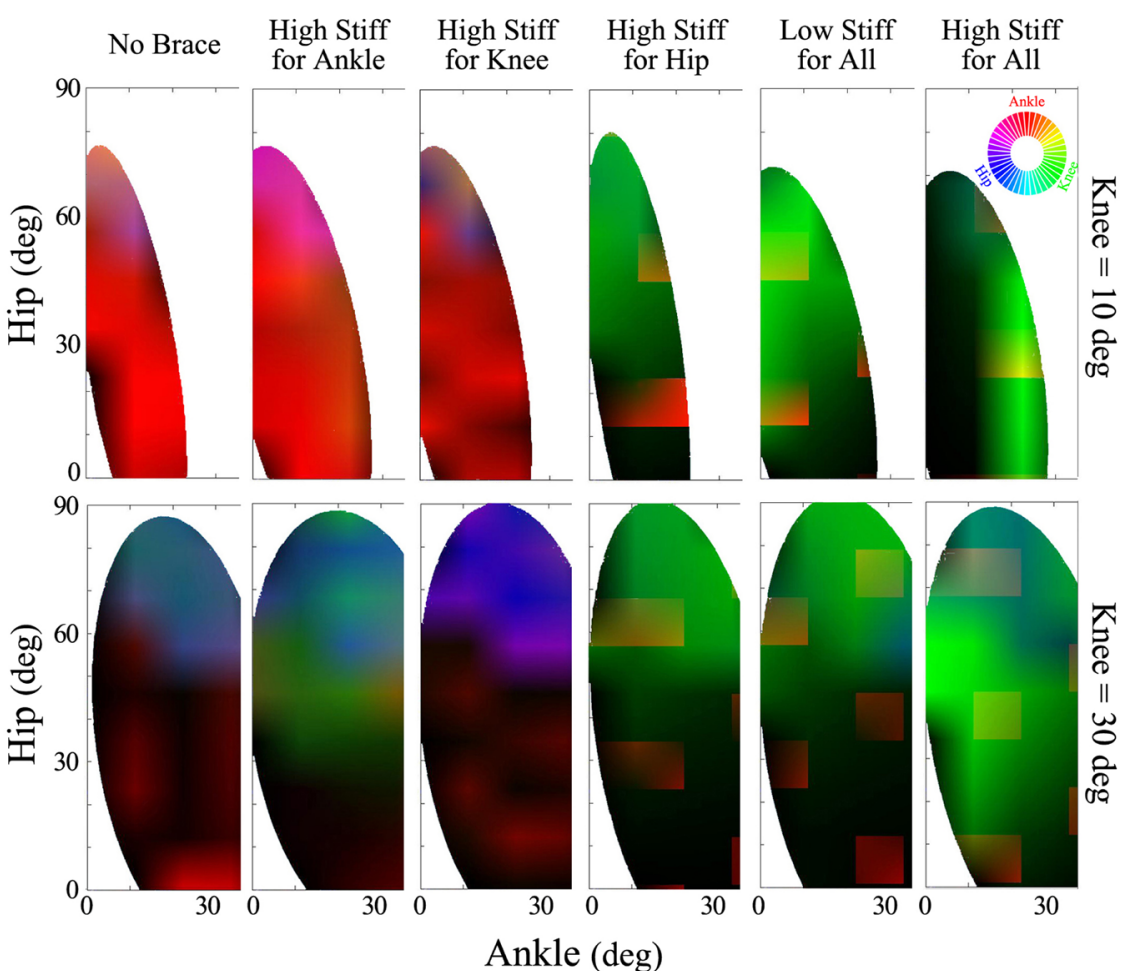

Figure 4. Color representation of the role-playing joint mechanisms in providing balance

The red-green-blue spectra illustrate the ankle, knee, and hip strategies, respectively

center of pressure post urography, which is inherently a dynamic behavior.

Addition of the braces to the joints assisted the supportive muscles acting on that joint. Without wearing the braces, the muscles acting on the joints tolerated the total shares of the weight moments; however, the braces can sustain some portions as a passive stiffness on the joints. Therefore, as depicted in Figure 2, the area trapped within the feasible postures expanded. This expansion meant that the body was able to provide the equilibrium in more postures.

The direction of expansion in the feasible ellipses was relatively along the axes that the braces were attached to the corresponded joints. For instance, when the brace was attached to the ankle joint, the ellipse expanded along the $\mathrm{x}$-axis, i.e. the ankle angle. It stated that wearing the ankle braces allowed the body to pose in new postures with more flexed ankle joint. Similarly, the hip braces empowered the body to provide the balance for more hip flexed postures; consequently, the feasible ellipse was elongated in the hip (y-axis) direction.

The expansion of the areas against the knee flexion angles was not linear. The midrange knee flexion had the highest area or number of feasible postures. This maximum area was for all combinations of wearing the braces and putting them did not change the case. However, there were some extra postures added to the feasible ones by wearing the braces. Among the single joint braces, the ankle brace wearing was the most effective one.

Recently, Webster et al. showed that more rigid braces for the patient with chronic ankle instability led to better standing indices [14]. Moreover, Ashtiani and Azghani indicated that the elders may use knee flexion to reduce the overall muscular effort (energy) and concluded that limiting the range of knee motion increased the total body effort [23] leading to fatigue. Therefore, external support of the ankle may be a more beneficial option. Also, since the ankle muscles, specifically the calf muscles that play a considerable role is providing the balance, were supported by brace stiffness aids, the body was allowed to sway more than the no brace condition.

It implied that more feasible postures could provide the equilibrium. The importance of ankle, however, originated from its weight bearing nature. If the body was modeled by a single inverted pendulum, total weight of the body should be traded off by the calf. Although it is not true to assume a single inverted pendulum for the 
body, the calf muscles tolerated a remarkable share of the weight moment and wearing the ankle braces can assist to add more feasible postures.

Joint contributions were changed by putting the braces. Without the braces, the ankle is the prominent mechanism to provide equilibrium in the lower knee angles. When the knee was remained extended, the ankle provided the balance and wearing the ankle and knee braces could not decrease its prominence. The knee brace attachment caused a slight decrease in the ankle role when the postures were in flexed hip positions. The blue spots on top of the feasible ellipse in 10-degree knee flexion showed the role-playing of the hip by the knee braces. It may be due to the effects of increase in knee tolerance.

The knee in aids of its brace sustained a share of the upper segment weight moment, and therefore, lower portions though slight remained for the ankle muscles, which was clearer in flexed hip postures. The knee bracing, however, was not led to considerable changes in the ankle or hip mechanism, since the knee angle was low in 10 degrees. When it flexed to 30 degrees, knee bracing played a more influential role. The ankle that is inferior to the knee relieved from burdening the total body weight and its role in provision of balance decreased. In contrast, the hip mechanism, specifically when it is more flexed, was about to counteract the upper body weight moment. Therefore, the blue spectrum was more observable in Figure 4.

The ankle bracing effect was relied on the knee angle. In low knee flexion (10 degrees), attachment of the ankle brace just relieved that the ankle could be recruited to provide the equilibrium. The postures that already stood by the ankle mechanism shared the efforts between the knee and hip joints.

The upper hip flexion caused hip muscle efforts and lower angles belonged to the knee mechanism. Hip bracing, on the other hand, resulted in the prominence of the knee mechanism. The dominant green color in the feasible ellipse with limited ankle (red color) role-playing showed that the attachment of hip transferred the weight moments to the knee. Although some muscles were biarticular between the knee and hip, tolerating the weight moment was possible by activation of the hamstrings.

In conclusion, the current study aimed at evaluating the effects of wearing joint braces using a numerical model of human standing. The model found the muscle activation based on optimization. Employment of the braces could sustain some shares of the body weight and changed the muscle recruitment patterns to provide the equilibrium. The knee mechanism played considerable role when braces were used for other joints. Attachment of the braces increased the body's ability to take more static postures. If only its allowed to wear only one brace, the ankle bracing could theoretically add more feasible balanced postures.

\section{Ethical Considerations}

\section{Compliance with ethical guidelines}

There was no ethical considerations to be considered in this research.

\section{Funding}

The present paper was extracted from the $\mathrm{PhD}$. thesis of the second author, in Faculty of Biomedical Engineering, Sahand University of Technology, Tabriz, Iran.

\section{Authors' contributions}

Conceptualization: Mahmood-Reza Azghani; Methodology: All authors; Investigation: All authors; Writingoriginal draft: Mohammed Ashtiani; Writing-review \& editing: Mohammed Ashtiani; Funding Acquisition: Mahmood-Reza Azghani; Resources: Mahmood-Reza Azghani; and Supervision: Mahmood-Reza Azghani.

\section{Conflict of interest}

The authors declared no conflict of interest.

\section{References}

[1] Mazaheri M, Coenen P, Parnianpour M, Kiers H, van Dieën $\mathrm{JH}$. Low back pain and postural sway during quiet standing with and without sensory manipulation: A systematic review. Gait \& Posture. 2013; 37(1):12-22. [DOI:10.1016/j.gaitpost.2012.06.013] [PMID]

[2] Ashtiani MN, Azghani MR. Nonlinear dynamics analysis of the human balance control subjected to physical and sensory perturbations. Acta Neurobiologiae Experimentalis. 2017; 77(2):168-75. [DOI:10.21307/ane-2017-049]

[3] Collins JJ, De Luca CJ. Open-loop and closed-loop control of posture: A random-walk analysis of center-of-pressure trajectories. Experimental Brain Research. 1993; 95(2):308-18. [DOI:10.1007/BF00229788]

[4] Horak FB, Kluzik J, Hlavacka F. Velocity dependence of vestibular information for postural control on tilting surfaces. Journal of Neurophysiology. 2016; 116(3):1468-79. [DOI:10.1152/jn.00057.2016] [PMID] [PMCID] 
[5] Billot M, Handrigan GA, Simoneau M, Corbeil P, Teasdale N. Short term alteration of balance control after a reduction of plantar mechanoreceptor sensation through cooling. Neuroscience Letters. 2013; 535:40-4. [DOI:10.1016/j.neulet.2012.11.022] [PMID]

[6] Chiarovano E, Wang W, Rogers SJ, MacDougall HG, Curthoys IS, De Waele C. Balance in virtual reality: Effect of age and bilateral vestibular loss. Frontiers in Neurology. 2017; 8:5. [DOI:10.3389/fneur.2017.00005] [PMID] [PMCID]

[7] Rubenstein LZ, Josephson KR. Risk factors for falls: A central role in prevention. Generations. 2002; 26(4):15.

[8] Papalia R, Franceschi F, Tecame A, D'Adamio S, Maffulli $\mathrm{N}$, Denaro V. Anterior cruciate ligament reconstruction and return to sport activity: Postural control as the key to success. International Orthopaedics. 2015; 39(3):527-34. [DOI:10.1007/ s00264-014-2513-9] [PMID]

[9] Negahban H, Mazaheri M, Kingma I, van Dieën JH. A systematic review of postural control during single-leg stance in patients with untreated anterior cruciate ligament injury. Knee Surgery, Sports Traumatology, Arthroscopy. 2014; 22(7):1491-504. [PMID]

[10] Suomi R, Koceja DM. Postural sway characteristics in women with lower extremity arthritis before and after an aquatic exercise intervention. Archives of Physical Medicine and Rehabilitation. 2000; 81(6):780-5. [DOI:10.1016/S00039993(00)90111-4] [PMID]

[11] Gauchard GC, Vançon G, Meyer P, Mainard D, Perrin PP. On the role of knee joint in balance control and postural strategies: Effects of total knee replacement in elderly subjects with knee osteoarthritis. Gait Posture. 2010; 32(2):155-60. [DOI:10.1016/j.gaitpost.2010.04.002] [PMID]

[12] Birmingham TB, Kramer JF, Kirkley A, Inglis JT, Spaulding SJ, Vandervoort AA. Knee bracing for medial compartment osteoarthritis: Effects on proprioception and postural control. Rheumatology. 2001; 40(3):285-9. [DOI:10.1093/rheumatology $/ 40.3 .285]$

[13] Pintsaar A, Brynhildsen J, Tropp H. Postural corrections after standardised perturbations of single limb stance: Effect of training and orthotic devices in patients with ankle instability. British Journal of Sports Medicine. 1996; 30(2):151-5. [DOI:10.1136/bjsm.30.2.151]

[14] Webster CA, Nussbaum MA, Madigan ML. Stiffness and proprioceptive contributions of ankle braces and the influence of localized muscle fatigue. Journal of Electromyography and Kinesiology. 2017; 34:37-43. [DOI:10.1016/j.jelekin.2017.02.009] [PMID]

[15] Hettich G, Assländer L, Gollhofer A, Mergner T. Human hip- ankle coordination emerging from multisensory feedback control. Human Movement Science. 2014; 37:123-46. [DOI:10.1016/j.humov.2014.07.004] [PMID]

[16] Chiba R, Takakusaki K, Ota J, Yozu A, Haga N. Human upright posture control models based on multisensory inputs; in fast and slow dynamics. Neuroscience Research. 2016; 104:96104. [DOI:10.1016/j.neures.2015.12.002] [PMID]

[17] Wang Z, Ko JH, Challis JH, Newell KM. The degrees of freedom problem in human standing posture: Collective and Component Dynamics. PlOS One. 2014; 9(1):e85414. [DOI:10.1371/journal.pone.0085414]
[18] Jeon HS, Hwang S, Woo YK. The effect of ankle and knee immobilization on postural control during standing. Knee. 2013; 20(6):600-4. [DOI:10.1016/j.knee.2012.09.001] [PMID]

[19] De Freitas PB, Freitas SM, Duarte M, Latash ML, Zatsiorsky VM. Effects of joint immobilization on standing balance. Human Movement Science. 2009; 28(4):515-28. [DOI:10.1016/j. humov.2009.02.001] [PMID] [PMCID]

[20] Kagaya H, Sharma M, Kobetic R, Marsolais EB. Ankle, knee, and hip moments during standing with and without joint contractures: Simulation study for functional electrical stimulation. American Journal of Physical Medicine \& Rehabilitation. 1998; 77(1):49-54. [DOI:10.1097/00002060199801000-00009]

[21] Ashtiani MN, Azghani MR. Predictive models for estimation of the human stance equilibrium parameters using inverse dynamics and response surface method. Journal of Musculoskeletal Research. 2017; 20(3):1750016. [DOI:10.1142/ S0218957717500166]

[22] Ashtiani MN, Azghani MR, Parnianpour M. Initial balance in human standing postures: Roles of the joint mechanisms. Proceedings of the Institution of Mechanical Engineers, Part H. 2018; 232(12):1255-60. [DOI:10.1177/0954411918811858]

[23] Ashtiani MN, Azghani MR. Effect of aging on feasibility and contribution of joint mechanisms in balanced standing using biomechanical modeling. Zahedan Journal of Research in Medical Sciences. 2017; 19(8):e11786. [DOI:10.5812/ zjrms.11786]

[24] Cambridge University Engineering Department. Materials data book. Cambridge: Cambridge University; 2003.

[25] Devita PA, Skelly WA. Effect of landing stiffness on joint kinetics and energetics in the lower extremity. Medicine \& Science in Sports \& Exercise. 1992; 24(1):108-15. [DOI:10.1249/00005768-199201000-00018] [PMID] 
This Page Intentionally Left Blank 